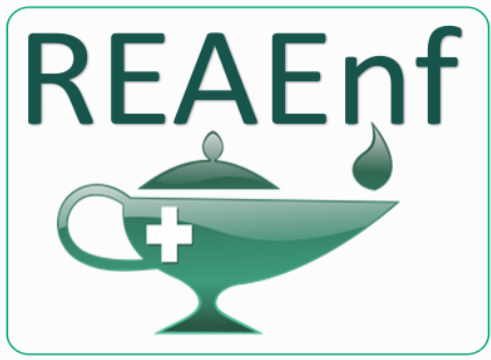

Revista Eletrônica Acervo Enfermagem
ARTIGO ORGINAL

Recebido em: 11/2019

Aceito em: $12 / 2019$

Publicado em: 2/2020

\title{
Perfil de nascimentos no estado de Minas Gerais no período de 2013 a 2017
}

\author{
Birth's profile in the state of Minas Gerais from 2013 to 2017
}

\section{Perfil de los nacimientos en el estado de Minas Gerais de 2013 a 2017}

Nara Pereira de Faria Carvalho de Alencar ${ }^{1 *}$, Jonathan Mendes de Castro, Ranyelli Estefany da Costa Martins ${ }^{1}$, Marcela Alves Azevedo ${ }^{1}$, Talles Vinícius de Castro Oliveira ${ }^{1}$, Enaile de Souza Proti $^{1}$, Diego Azevedo Araújo ${ }^{1}$, Jacqueline Souza Dutra Arruda ${ }^{1}$, Carlos Henrique Wernersbach Guerra $^{1}$, Wendel Jose Teixeira Costa ${ }^{1,2}$.

\begin{abstract}
Resumo: Esse trabalho tem como objetivo descrever o perfil epidemiológico dos Nascidos Vivos por raça/cor das mães, no Estado de Minas Gerais, no período de 2013 a 2017. Trata-se de estudo transversal descritivo, com utilização dados de natureza secundária oriundos do Sistema de Informação Nascidos Vivos (SINASC) do Departamento de Informática do Sistema Único de Saúde (DATASUS). A amostra foi composta de 1.223.629 nascidos vivos residentes no Estado de Minas Gerais, cadastrados no SINASC, no período 2013 a 2017. Dentre estes, 435.493 (35,6\%) nasceram de mulheres da raça Branca, 777.800.364 $(63,6 \%)$ de mulheres da raça Preta e Parda e $10.336(0,8 \%)$ de mulheres da raça Amarela e Indígena. No período analisado, o perfil epidemiológico nos nascimentos no estado de Minas Gerais, caracteriza-se por maioria do sexo masculino, de mães pretas e pardas, nas faixas etárias entre 20 e 34 anos, com escolaridade entre 8 e 12 anos de estudo, com companheiro. Maioria dos nascimentos à termo, com prénatal adequado em número de consultas, por parto cesáreo, índice de Apgar no 1ํㅡ e 5ํㅡㄴ mutos acima de 8, com peso adequado ao nascer.
\end{abstract}

Palavras-chave: Nascido vivo, Perfil epidemiológico, Saúde da mulher.

Abstract: This paper aims to describe the epidemiological profile of live births by race / color of mothers, in the state of Minas Gerais, from 2013 to 2017. This is a descriptive cross-sectional study, using secondary data from of the Live Birth Information System - SINASC of the Department of Informatics of the Unified Health System - DATASUS. The sample consisted of 1,223,629 live births residing in the State of Minas Gerais, registered in the SINASC, from 2013 to 2017. Among these, 435,493 (35.6\%) were born to White women, $777,800,364(63.6 \%)$ of Black and Parda women and 10,336 (0.8\%) of Yellow and Indigenous women. In the period analyzed, the epidemiological profile of births in the state of Minas Gerais is characterized by the majority of males, black and brown mothers, aged between 20 and 34 years, with education between 8 and 12 years of study, with companion. Most term births, with adequate prenatal care in number of consultations, by cesarean section, Apgar score at 1 and 5 minutes above 8 , with adequate birth weight.

Keywords: Live birth, Health profile, Women's health.

${ }^{1}$ Centro Universitário de Caratinga (UNEC), Caratinga-MG. *E-mail: narapfcalencar@gmail.com 2Laboratório de Escrita Científica, Escola Superior de Ciências da Santa Casa de Misericórdia (EMESCAM), Vitória-ES. 
Resumen: Este artículo tiene como objetivo describir el perfil epidemiológico de los nacidos vivos por raza / color de las madres, en el estado de Minas Gerais, de 2013 a 2017. Este es un estudio descriptivo de corte transversal, que utiliza datos secundarios de del Sistema de Información de Nacimientos Vivos - SINASC del Departamento de Informática del Sistema Único de Salud - DATASUS. La muestra consistió en $1,223,629$ nacidos vivos que residen en el Estado de Minas Gerais, registrados en el INASC, de 2013 a 2017. Entre estos, 435,493 (35.6\%) nacieron de mujeres blancas, $777,800,364(63.6 \%)$ de mujeres negras y pardas y $10,336(0.8 \%)$ de mujeres amarillas e indígenas. En el período analizado, el perfil epidemiológico de los nacimientos en el estado de Minas Gerais se caracteriza por la mayoría de los hombres, madres negras y marrones, con edades entre 20 y 34 años, con educación entre 8 y 12 años de estudio, con compañero La mayoría de los nacimientos a término, con visitas prenatales adecuadas, cesárea, puntaje de Apgar a 1 y 5 minutos por encima de 8 , con peso de nacimiento adecuado.

Palabras clave: Nacido vivo, Perfil de salud, Salud de la mujer.

\section{INTRODUÇÃO}

A gestação é um período de transformações no corpo e também no emocional da mulher. Essas transformações são individuais e vivenciadas de forma única de gestante para gestante. Sendo a primeira vez ou não que a mulher vivencia a gravidez, os sentimentos de medo, angústia e de alegria podem estar presentes, assim como a dúvida. Existe também o aspecto cultural, como o medo das mudanças no corpo e o medo do parto (MOTTA EM, et al., 2016).

Um recém-nascido $(\mathrm{RN})$ sadio sem trauma para a mãe é o que espera de uma gestação. Porém, devido a algumas situações que geram complicações durante a evolução da gravidez, no parto ou com o concepto, por vezes isso não é possível. O que gera riscos à saúde materna e do concepto (BRASIL, 2006).

As políticas públicas de atenção à saúde da mulher no ciclo gravídico-puerperal estão fundamentadas no planejamento familiar, na atenção pré-natal, parto e puerpério, tendo como objetivo principal a redução da morbimortalidade materna e neonatal com atendimento qualificado, humanizado, garantia de acesso integral à consultas, exames, imunização, procedimentos, educação em saúde, com condutas acolhedoras e livre de intervenções desnecessárias em todos os níveis de atenção à saúde (BRASIL, 2006).

$\mathrm{Na}$ busca pela melhoria da assistência materno infantil, o Ministério da Saúde lançou, em 2011, o programa Rede Cegonha. O Rede Cegonha é uma importante estratégia do Governo Federal, uma vez que objetiva a implementação de um novo modelo de atenção à saúde da mulher e da criança. Essa estratégia tem como objetivo aplicar um novo modelo de atenção à saúde da mulher e da criança, com foco no parto, nascimento, crescimento e desenvolvimento da criança, garantia de acesso, acolhimento e resolutividade e a redução da taxa de mortalidade materna e neonatal (CAVALCANTI PCS, 2010; BRASIL, 2011).

Conhecer os determinantes de saúde de uma população é de fundamental importância para o planejamento e a implementação de estratégias efetivas de melhoria das condições de saúde. Conhecer o perfil dos nascidos vivos, traçados em tempo e região permitem um conhecimento epidemiológico e demográfico, o que contribui para um melhor planejamento por parte dos gestores públicos, possibilitando desenvolver estratégias efetivas para melhorar as condições de saúde da população (FRICHE AAL, et al., 2006).

Caracterizam-se como determinantes sociais em saúde os fatores sociais, econômicos, culturais, étnicos/raciais, psicológicos e comportamentais que influenciam a ocorrência de problemas de saúde e seus fatores de risco na população". As relações entre determinantes sociais e saúde consistem em estabelecer uma hierarquia de determinações entre fatores mais distais, sociais, econômicos e políticos e mais proximais relacionados diretamente ao modo de vida, sendo distintos os fatores que afetam a situação de saúde de grupos e de pessoas (BUSS PM e PELLEGRINI FILHO A, 2007).

No ano de 1990 o Ministério da Saúde (MS) instituiu no Brasil o Sistema de Informações sobre Nascidos Vivos (SINASC), que é um sistema que registra todos os dados associados aos nascidos vivos, desempenhando vital papel no que se trata à estatística, epidemiologia e demografia. O registro no SINASC 
é realizado à partir da Declaração de Nascidos Vivos (DN) que é um documento padronizado no Brasil, preenchido para todos os Recém-nascidos (RN) no país (MELLO JORGE MHP, et al., 1992).

Por intermédio desses registros é possível subsidiar intervenções relacionadas à saúde da mulher e da criança para todos os níveis de atenção do Sistema Único de Saúde (SUS), especialmente, ações de atenção à gestante e ao recém-nascido (RN), pois são registrados nas (DN), dados sobre a mãe e o recémnascido, compondo o perfil epidemiológico dos nascimentos. Além disto, o acompanhamento da evolução das séries históricas do (SINASC) permite a identificação de prioridades de intervenção, o que contribui para efetiva melhoria do sistema (BRASIL, 2017).

$\mathrm{Na}$ DN consta dados referentes ao RN como também dados sobre a mãe, como escolaridade e idade; informações sobre o pré-natal, sendo o número de consultas realizadas um dos registros; a idade gestacional e as informações do parto, como via de parto, Apgar e peso ao nascer. O registro desses dados permite uma análise estatística detalhada, o que permite conhecer aspectos socioeconômicos da mãe e evidencia a qualidade da assistência à saúde na atenção pré-natal e ao RN (MELLO JORGE MHP, et al., 1992).

Frente ao exposto acima, esse trabalho tem como objetivo descrever o perfil epidemiológico dos Nascidos Vivos por raça/cor das mães, no Estado de Minas Gerais, no período de 2013 a 2017.

\section{MÉTODOS}

Trata-se de estudo transversal descritivo, com utilização dados de natureza secundária oriundos do Sistema de Informação Nascidos Vivos - SINASC do Departamento de Informática do Sistema Único de Saúde - (DATASUS).

A população do estudo foi constituída de nascidos vivos, filhos de mães residentes no Estado de Minas Gerais no período de 2013 a 2017.

Os dados foram extraídos do Sistema de Informação Nascidos Vivos o - Sinasc, fornecido pelo Departamento de Informática do Sistema Único de Saúde - DATASUS, website: www.datasus.gov.br e organizados em arquivo de planilhas do software Microsoft ${ }^{\circledR}$ Office Excel ${ }^{\circledR}$ versão 15.0, por dois pesquisadores independentes com o propósito de identificar possíveis discrepâncias.Realizou-se uma análise exploratória para reconhecimento das variáveis e correção de possíveis erros ou inconsistências na digitação dos dados. Feitas as correções necessárias precedeu-se a organização e análise dos dados, aplicando-se a estatística descritiva.

O SINASC propicia um aporte significativo de dados sobre nascidos vivos, com suas características mais importantes, como sexo, local onde ocorreu o nascimento, tipo de parto e peso ao nascer, entre outras. A base de dados nacional é gerada pelo DATASUS em cooperação com o Sistema de Vigilância Sanitária.

O presente estudo envolveu apenas a descrição e análise de dados secundários de população e nenhuma informação com identificação individual foi obtida para a realização deste estudo. Por se tratar de utilização de dados secundários não houve necessidade de aprovação do comitê de ética.

\section{RESULTADOS}

A amostra do presente estudo foi composta por um total de 1.223 .629 nascidos vivos residentes no Estado de Minas Gerais, cadastrados no Sistema de Informações sobre Nascidos Vivos- SINASC, no período 2013 a 2017. Dentre estes, 435.493 (35,6\%) nasceram de mulheres da raça Branca, 777.800.364 $(63,6 \%)$ de mulheres da raça Preta e Parda e 10.336 (0,8\%) de mulheres da raça Amarela e Indígena.

\section{Perfil demográfico materno}

Ao analisar o sexo prevalência ligeiramente maior foi vista no sexo masculino em todas as raças analisadas, sendo 50,9\% ( $n=221.784), 51,2 \%(n=398.559)$ e 50,9\% ( $n=5.259)$ nas raças Branca, Preta e Parda e Amarela e Indígena respectivamente. Maior prevalência de nascidos vivos foi vista na faixa etária 
materna de 20 a 34 anos em todas as raças analisadas, sendo $70,6 \%$ ( $n=307.673$ ) na raça Branca, 69,5\% $(n=540.950)$ nas raças Preta e Parda e 70,7\% ( $n=7.307)$ nas raças Amarela e Indígena. Quanto à instrução da mãe maior prevalência foi vista na instrução de 8 a 12 anos de estudo em todas as raças analisadas, sendo $84,7 \%(n=368.771), 75,5 \%(n=587.574)$ e $73,9 \%(n=7.640)$ nas raças Branca, Preta e Parda e Amarela e Indígena respectivamente. Ao analisar o estado civil das mulheres maior prevalência foi vista em mulheres com companheiro em todas as raças analisadas, sendo 66,9\% ( $n=291.476), 52,8 \%(n=410.588)$ e $58,0 \%$ ( $n=6.000)$ nas raças Branca, Preta e Parda e Amarela e Indígena respectivamente.

A Tabela 1 apresenta o perfil demográfico materno, analisando idade, instrução e estado civil da mãe por raça.

Tabela 1 - População e características demográficas.

\begin{tabular}{|c|c|c|c|c|c|c|c|c|}
\hline & \multicolumn{2}{|c|}{ Branca } & \multicolumn{2}{|c|}{ Preta e Parda } & \multicolumn{2}{|c|}{ Amarela e Indígena } & \multicolumn{2}{|c|}{ Total } \\
\hline & $\mathbf{n}$ & $\%$ & $\mathbf{n}$ & $\%$ & $\mathbf{n}$ & $\%$ & $\mathbf{n}$ & $\%$ \\
\hline Amostra & 435.493 & 35,6 & 777.800 & 63,6 & 10.336 & 0,8 & 1.223 .629 & 100 \\
\hline \multicolumn{9}{|l|}{ Sexo } \\
\hline Masculino & 221.784 & 50,9 & 398.559 & 51,2 & 5.259 & 50,9 & 625.602 & 51,1 \\
\hline Feminino & 213.640 & 49,1 & 379.127 & 48,7 & 5.074 & 49,1 & 597.841 & 48,9 \\
\hline Ignorado & 69 & 0,0 & 114 & 0,0 & 3 & 0,0 & 186 & 0,0 \\
\hline \multicolumn{9}{|l|}{ Idade } \\
\hline Até 14 anos & 1.712 & 0,4 & 5.377 & 0,7 & 94 & 0,9 & 7.183 & 0,6 \\
\hline 15 a 19 anos & 488.54 & 11,2 & 129.657 & 16,7 & 1.640 & 15,9 & 180.151 & 14,7 \\
\hline 20 a 34 anos & 307.673 & 70,6 & 540.950 & 69,5 & 7.307 & 70,7 & 855.930 & 70,0 \\
\hline 35 a 49 anos & 77.202 & 17,7 & 101.727 & 13,1 & 1.294 & 12,5 & 180.223 & 14,7 \\
\hline $50+$ & 52 & 0,0 & 88 & 0,0 & 1 & 0,0 & 141 & 0,0 \\
\hline \multicolumn{9}{|l|}{ Instrução } \\
\hline Nenhum & 606 & 0,01 & 2.754 & 0,4 & 484 & 4,7 & 3.844 & 0,3 \\
\hline Até 7 anos & 55.570 & 12,8 & 173.244 & 22,3 & 2.015 & 19,5 & 230.829 & 18,9 \\
\hline 8 a 12 anos & 368.771 & 84,7 & 587.574 & 75,5 & 7.640 & 73,9 & 963.985 & 78,8 \\
\hline Ignorado & 10.546 & 2,4 & 14.228 & 1,8 & 197 & 1,9 & 24.971 & 0,2 \\
\hline \multicolumn{9}{|l|}{ Estado civil } \\
\hline Sem companheiro & 141.154 & 32,4 & 361.779 & 46,5 & 4243 & 41,1 & 507.176 & 41,4 \\
\hline Com companheiro & 291.476 & 66,9 & 410.588 & 52,8 & 6.000 & 58,0 & 708.064 & 57,9 \\
\hline Ignorado & 2.863 & 0,7 & 5.433 & 0,7 & 93 & 0.9 & 8.389 & 0,7 \\
\hline
\end{tabular}

Fonte: SINASC/DATASUS, 2019

\section{Duração da gestação, consultas pré-natal e desfechos obstétricos}

Quanto à duração da gestação maior prevalência foi vista na gestação à termo (37 a 41 semanas) em todas as raças analisadas, sendo $84,9 \%(n=369.900)$ na raça Branca, $83,4 \%(n=648.637)$ nas raças Preta $e$ Parda e $82,7 \%$ ( $n=8.552)$ nas raças Amarela e Indígena. Quanto ao número de consultas pré-natal maior prevalência foi vista de 7 ou mais consultas em todas as raças analisadas, sendo $80,6 \%$ ( $n=350.980)$, $71,1 \%(n=553.321)$ e $70,5 \%(n=7.284)$ nas raças Branca, Preta e Parda e Amarela e Indígena respectivamente. Ao analisar a vai de parto maior prevalência no parto cesáreo na raça Branca e nas raças Preta e Parda, sendo 70,0\% ( $n=304.719)$ e $50,3 \%(n=391.120)$ respectivamente. Nas raças Amarela e Indígena houve maior prevalência na via de parto vaginal, sendo $52,6 \%(n=5.436)$. Ao analisar o Apgar, avaliado no $1^{\circ}$ e no $5^{\circ}$ minuto, em ambos maior prevalência foi vista no índice de 8 a 10 em todas as raças analisadas, sendo $87,6 \%(n=381.614), 84,8 \%(n=659.599)$ e $79,9 \%(n=8.263)$ nas raças Branca, Preta e Parda e Amarela e Indígena respectivamente no Apgar $1^{\circ}$ minuto, e 95,9\% ( $\left.n=417.795\right), 94,4 \%(n=734.114)$ e 89,0\% ( $n=9.198)$ nas raças Branca, Preta e Parda e Amarela e Indígena respectivamente no Apgar $5^{\circ}$ minuto. Ao analisar o peso ao nascer maior prevalência foi vista no Peso adequado (3.000 a 3.999g), sendo $62,3 \%(n=271.164), 61,2 \%(n=476.016)$ e 61,2\% $(n=6.322)$ nas raças Branca, Preta e Parda e Amarela e Indígena respectivamente. 
A Tabela 2 apresenta duração da gestação, pré-natal e os desfechos obstétricos, analisando via de parto, apgar $1^{\circ}$ e $5^{\circ}$ minuto, e peso ao nascer.

Tabela 2 - Desfechos obstétricos.

\begin{tabular}{|c|c|c|c|c|c|c|c|c|}
\hline & \multicolumn{2}{|c|}{ Branca } & \multicolumn{2}{|c|}{ Preta e Parda } & \multicolumn{2}{|c|}{ Amarela e Indígena } & \multicolumn{2}{|c|}{ Total } \\
\hline & $\mathbf{n}$ & $\%$ & $\mathbf{n}$ & $\%$ & $n$ & $\%$ & $\mathbf{n}$ & $\%$ \\
\hline Amostra & 435.493 & 35,6 & 777.800 & 63,6 & 10.336 & 0,8 & 1.223 .629 & 100 \\
\hline \multicolumn{9}{|l|}{ Duração da gestação } \\
\hline Pré-termo & 47.735 & 11,0 & 87.939 & 11,3 & 1.207 & 11,7 & 136.881 & 11,2 \\
\hline À termo & 369.900 & 84,9 & 648.637 & 83,4 & 8.552 & 82,7 & 1.027 .089 & 83,9 \\
\hline Pós-termo & 9.116 & 2,1 & 23.337 & 3,0 & 283 & 2,7 & 32.736 & 2,7 \\
\hline Ignorado & 8.742 & 2,0 & 17.887 & 2,3 & 294 & 2,8 & 26.923 & 2,2 \\
\hline \multicolumn{9}{|l|}{ Consulta Pré-natal } \\
\hline Nenhuma & 4.280 & 1,0 & 10.221 & 1,3 & 137 & 1,3 & 14.638 & 1,2 \\
\hline De 1 a 3 consultas & 11.109 & 2,6 & 36.320 & 4,7 & 531 & 5,1 & 47.960 & 3,9 \\
\hline De 4 a 6 consultas & 67.779 & 15,6 & 174.785 & 22,5 & 2.347 & 22,7 & 244.911 & 20,0 \\
\hline 7 ou mais consultas & 350.980 & 80,6 & 553.321 & 71,1 & 7.284 & 70,5 & 911.585 & 74,5 \\
\hline Ignorado & 1.345 & 0,3 & 3.153 & 0,4 & 37 & 0,4 & 4.535 & 0,4 \\
\hline \multicolumn{9}{|l|}{ Via de parto } \\
\hline Vaginal & 129.918 & 23,0 & 385.109 & 49,5 & 5.436 & 52,6 & 520.463 & 42,5 \\
\hline Cesáreo & 304.719 & 70,0 & 391.120 & 50,3 & 4.884 & 47,3 & 700.723 & 57,3 \\
\hline Ignorado & 856 & 0,2 & 1.571 & 0,2 & 16 & 0,2 & 2.443 & 0,2 \\
\hline \multicolumn{9}{|l|}{ Apgar $1^{\circ}$ min } \\
\hline Até 7 & 43.960 & 10,1 & 91.336 & 11,7 & 1.141 & 11,0 & 136.437 & 11,2 \\
\hline 8 a 10 & 381.614 & 87,6 & 659.599 & 84,8 & 8.263 & 79,9 & 1.049 .476 & 85,8 \\
\hline Ignorado & 9.919 & 2,3 & 26.865 & 3,5 & 932 & 9,0 & 37.716 & 3,1 \\
\hline \multicolumn{9}{|l|}{ Apgar 5 $\mathrm{min}$} \\
\hline Até 7 & 7.932 & 1,8 & 17.357 & 2,2 & 229 & 2,2 & 25.518 & 2,1 \\
\hline 8 a 10 & 417.795 & 95,9 & 734.114 & 94,4 & 9.198 & 89,0 & 1.161 .107 & 94,9 \\
\hline Ignorado & 9.766 & 2,2 & 26.329 & 3,4 & 909 & 8,8 & 37.004 & 94,9 \\
\hline \multicolumn{9}{|l|}{ Peso ao nascer (g) } \\
\hline Baixo peso $(<2.500)$ & 39.401 & 9,0 & 73.933 & 9,5 & 996 & 9,6 & 114.330 & 9,3 \\
\hline $\begin{array}{l}\text { Peso insuficiente } \\
(2.500 \text { a } 2.999)\end{array}$ & 110.788 & 25,4 & 197.259 & 25,4 & 2.637 & 25,5 & 310.684 & 25,4 \\
\hline $\begin{array}{l}\text { Peso adequado ( } 3.000 \\
\text { a } 3.999)\end{array}$ & 271.164 & 62,3 & 476.016 & 61,2 & 6.322 & 61,2 & 753.502 & 61,6 \\
\hline $\begin{array}{l}\text { Excesso de peso } \\
\text { ( } 4.000 \text { ou mais) }\end{array}$ & 14.125 & 3,2 & 30.494 & 3,9 & 378 & 3,7 & 44.997 & 3,7 \\
\hline Ignorado & 15 & 0,0 & 98 & 0,0 & 3 & 0,0 & 116 & 0,0 \\
\hline
\end{tabular}

Fonte: SINASC/DATASUS, 2019.

\section{DISCUSSÃO}

Ao analisar o sexo, maior prevalência foi vista no sexo masculino em todas as raças analisadas, sendo $50,9 \%(n=221.784), 51,2 \%$ ( $n=398.559)$ e 50,9\% ( $n=5.259)$ nas raças Branca, Preta e Parda e Amarela e Indígena respectivamente. Os valores se assemelham à um estudo de 2012 realizado em Serra-ES que analisou o perfil de nascimentos no município no período de 2001 a 2005 , registrando $51,4 \%$ dos nascidos vivos do sexo masculino (LIMA EFA, et al., 2012).

Maior prevalência de nascidos vivos foi vista na faixa etária materna de 20 a 34 anos em todas as raças analisadas, sendo 70,6\% ( $n=307.673)$ na raça Branca, 69,5\% $(n=540.950)$ nas raças Preta e Parda e 70,7\% $(n=7.307)$ nas raças Amarela e Indígena. Estudo realizado em Aracaju SE em 2012, que teve como objetivo 
de caracterizar o perfil dos nascidos vivos residentes no município de Aracaju no ano de 2010, converge com esse estudo, pois observou taxa de $72,14 \%$ das puérperas nas faixas etárias de 20 a 34 anos (CRAVO EO e OLIVEIRA JVR, 2012).

Estudo realizado no município de Maringá-PR em 2015 teve por objetivo descrever o perfil sociodemográfico e obstétrico das puérperas residentes em Maringá, atendidas pela Rede Mãe Paranaense e encontrou taxa de 72,5\% de puérperas nas faixas etárias de 20 a 34 anos (NOVAES ES, et al., 2015).

Quanto à instrução da mãe maior prevalência foi vista na instrução de 8 a 12 anos de estudo em todas as raças analisadas, sendo $84,7 \%(n=368.771), 75,5 \%(n=587.574)$ e $73,9 \%(n=7.640)$ nas raças Branca, Preta e Parda e Amarela e Indígena respectivamente.

Este estudo diverge de estudo realizado no município de Maranguape-CE em 2007 que descreveu o perfil dos nascidos vivos no município no período de 2000 a 2003 que observou maior prevalência em mulheres com instrução de 4 a 7 anos de estudo, registrando no ano de 2000 49,9\% e ano de 2003 39,4\% (OLIVEIRA NC, et al., 2007). Ao analisar o estado civil das mulheres maior prevalência foi vista em mulheres com companheiro em todas as raças analisadas, sendo 66,9\% ( $n=291.476), 52,8 \%(n=410.588)$ e $58,0 \%(n=6.000)$ nas raças Branca, Preta e Parda e Amarela e Indígena respectivamente.

Estudo realizado no município de Serra-ES em 2007, que descreveu o perfil social e obstétrico das puérperas usuárias do serviço de saúde de uma maternidade municipal no ano de 2002 revelou uma maior prevalência de puérperas com companheiro, sendo casadas $23,45 \%$; e em união consensual $48,01 \%$, concordando com o estudo atual nas taxas de puérperas com companheiros serem maiores do que as puérperas sem companheiro (PRIMO CC, et al., 2007).

Quanto à duração da gestação o estudo demonstrou maior prevalência na gestação à termo (37 a 41 semanas) em todas as raças analisadas, sendo $84,9 \%$ ( $n=369.900)$ na raça Branca, $83,4 \%$ ( $n=648.637)$ nas raças Preta e Parda e $82,7 \%$ ( $n=8.552)$ nas raças Amarela e Indígena. Convergindo com estudo realizado em Belo Horizonte em 2018, que descreveu o perfil epidemiológico dos nascidos vivos no município no período de 1994 a 2014, que constou 90,2\% das gestações à termo (FERREIRA VA, et al., 2018).

Quanto ao número de consultas pré-natal a maioria teve 7 ou mais consultas em todas as raças analisadas, sendo $80,6 \%(n=350.980), 71,1 \%(n=553.321)$ e $70,5 \%(n=7.284)$ nas raças Branca, Preta e Parda e Amarela e Indígena respectivamente. Estudo realizado no Piauí realizado em 2017 diverge do encontrado neste. Embora maior prevalência também foi vista na assistência em 7 ou mais consultas a taxa foi de apenas 45,8\% (DOS SANTOS GM, et al., 2017).

O presente estudo demonstrou um predomínio do parto cesáreo, acima do indicado pelo Organização Mundial de Saúde (OMS), que é de 15\%. O estudo evidenciou que no período de 2013 a 2017 no estado de Minas Gerais o parto cesáreo foi predominante na raça Branca e nas raças Preta e Parda, representando $70,0 \%$ e $50,3 \%$ dos partos respectivamente. Nas raças Amarela e Parda, embora o parto vaginal seja predominante, a taxa de cesáreas é elevada, sendo $47,3 \%$ dos partos.

Estudo semelhante foi realizado em Viçosa-MG em 2010, onde foi descrito o perfil dos nascidos vivos no município de Viçosa no período de 2001 a 2007, que registrou que 63,3\% dos partos do ano de 2007 foram cesáreas (PEREIRA JÚNIOR AC e HENRIQUES BD, 2010).

Segundo a OMS (2015) a taxa de cesáreas, a nível populacional, com índices superiores a 10\% não está associada à redução de mortalidade materna e neonatal. Estudo realizado no estado do Piauí em 2017, registrou uma taxa de $53,6 \%$ dos partos por via Vaginal, porém com $46,1 \%$ dos partos por via Cesárea, ultrapassando também o valor indicado pela OMS (DOS SANTOS GM, et al., 2017).

Ao analisar o índice de Apgar, avaliado no $1^{\circ}$ e no $5^{\circ}$ minuto, este estudo demonstrou que em ambos maior prevalência foi vista no índice de 8 a 10 em todas as raças analisadas, sendo $87,6 \%$ ( $n=381.614)$, $84,8 \%(n=659.599)$ e $79,9 \%(n=8.263)$ nas raças Branca, Preta e Parda e Amarela e Indígena respectivamente no Apgar $1^{\circ}$ minuto, e 95,9\% ( $\left.n=417.795\right), 94,4 \%(n=734.114)$ e $89,0 \%(n=9.198)$ nas raças Branca, Preta e Parda e Amarela e Indígena respectivamente no do 5ํㅡㄴ minuto. 
Estudo semelhante, realizado no município de Viçosa-MG, que descreveu o perfil dos nascidos vivos no município no período de 2001 a 2007, registrou índice de Apgar acima de 7 em 70,3\% em 2001 e 73,0\% em 2007 no $1^{\circ}$ minuto; e 80,0\% em 2001 e 89,0\% em 2007 no $5^{\circ}$ minuto (PEREIRA JÚNIOR AC e HENRIQUES BD, 2010).

Os valores se assemelham à um estudo realizado em Belo Horizonte em 2018, que descreveu o perfil epidemiológico dos nascidos vivos no município no período de 1994 a 2014. Esse estudou evidenciou uma taxa de $85,0 \%$ do índice entre 8 e 10 no Apgar no $1^{\circ}$ minuto e $95,4 \%$ no do $5^{\circ}$ minuto (FERREIRA VA, et al., 2018).

Ao avaliar o peso ao nascer o presente estudo evidenciou uma maior prevalência de nascidos vivos com peso adequado (3.000 a $3.999 \mathrm{~g})$ em todas as raças analisadas, sendo 62,3\% ( $\mathrm{n}=271.164), 61,2 \%$ $(n=476.016)$ e 61,2\% ( $n=6.322)$ nas raças Branca, Preta e Parda e Amarela e Indígena respectivamente.

Estudo realizado Foz do Iguaçu converge com os achados neste. Realizado no ano de 2010, avaliou o perfil epidemiológico de nascimentos no município de Foz do Iguaçu no período de 2000 a 2008. Houve predomínio, de $71,29 \%$ dos nascidos vivos com registro de peso acima de $3.000 \mathrm{~g}$ (RODRIGUES KSF e ZAGONEL IPS, 2010).

\section{CONCLUSÃO}

No período analisado, o perfil epidemiológico nos nascimentos no estado de Minas Gerais, caracterizase por maioria do sexo masculino, de mães pretas e pardas, nas faixas etárias entre 20 e 34 anos, com escolaridade entre 8 e 12 anos de estudo, com companheiro. Maioria dos nascimentos à termo, com prénatal adequado em número de consultas, por parto cesáreo, índice de Apgar no 1ํㅡ e 5ํㅡㄴ mutos acima de 8 , com peso adequado ao nascer.

\section{REFERÊNCIAS}

1. BRASIL, Ministério da Saúde, Secretaria de Atenção à Saúde. Pré-natal e puerpério: atenção qualificada e humanizada - manual técnico. Brasília (DF):Ministério da Saúde; 2006. 163 p.

2. CRAVO EO, OLIVEIRA JVR. Perfil epidemiológico dos nascidos vivos no município de Aracaju - Sergipe, Brasil. Idéias \& Inovação, 2012; 1(1): 9-17.

3. DOS SANTOS, GM et al. Análise do perfil das puérperas e dos nascidos vivos em um estado do nordeste brasileiro. Revista Uningá review, [S.I.], 2017; 31(1): 12-18.

4. FERREIRA VA, et al. Perfil Epidemiológico dos Nascidos Vivos em Belo Horizonte, MG, Brasil. Revista de Enfermagem do Centro-Oeste Mineiro, 2018; 8(2550).

5. FRICHE AAL, et al. Indicadores de saúde materno infantil em Belo Horizonte, Minas Gerais, Brasil, 2001: análise dos diferenciais intra-urbanos. Cad. Saúde Pública [online]. 2006; 22(9): 1955-1965.

6. LIMA EFA, et al. Perfil de nascimentos de um município: um estudo de coorte. Revista Brasileira de Pesquisa em Saúde, 2012; 14(1): 12-18.

7. MELLO JORGE MHP,et al. O sistema de informação sobre nascidos vivos-SINASC. São Paulo: Centro Brasileiro para Classificação de Doenças. Núcleo de Estudos em População e Saúde. Universidade de São Paulo; 1992.

8. MOTA EM, et al. Sentimentos e expectativas vivenciados pelas primigestas adolescentes com relação ao parto. Rev RENE [Internet], 2011; 12(4): 692-8.

9. NOVAES ES, et al. Perfil obstétrico de usuárias do Sistema Único de Saúde após implantação da Rede Mãe Paranaense. Ciênc Cuid Saúde, 2015; 14(4): 1436-44.

10. OLIVEIRA NC, et al. Perfil dos nascidos vivos de Maranguape-CE: a partir da série histórica de 2000 a 2003. Revista da Rede de Enfermagem do Nordeste, 2007; 8(1): 52-59.

11. PEREIRA JÚNIOR AC, HENRIQUES BD. Perfil dos nascidos vivos em Viçosa, Minas Gerais, no período de 2001 a 2007. Rev Méd Minas Gerais, 2010; 20(4): 508-513.

12. PRIMO CC, et al. Perfil social e obstétrico das puérperas de uma maternidade. Rev Enf UERJ, 2007; $15(2): 161-7$.

13. RODRIGUES KSF, et al. Perfil epidemiológico de nascimentos em Foz do Iguaçu/PR: indicador para planejamento do cuidado do enfermeiro. Esc. Anna Nery, Rio de Janeiro, 2010; 14(3): 534-542. 